\title{
ARTÍCULOS
}

\section{CLASE Y ACTIVISMO URBANO. PROPUESTAS A PARTIR DE UNA INVESTIGACIÓN ETNOGRÁFICA}

\section{CLASS AND URBAN ACTIVISM. LESSONS FROM AN ETHNOGRAPHIC RESEARCH}

\author{
José Antonio Mansilla López ${ }^{1}$
}

Observatori d'Antropologia del Conflicte Urbà (OACU)

Recibido: 17 de febrero de 2020; Aprobado: 3 de marzo de 2021.

Cómo citar este artículo / Citation: Mansilla López, José Antonio. 2021. "Clase y activismo urbano. Propuestas a partir de una investigación etnográfica”. Disparidades. Revista de Antropología 76(2): e018. doi: <https://doi.org/10.3989/dra.2021.018>.

RESUMEN: En la actualidad, las ciudades juegan un papel relevante en las dinámicas de acumulación del capital. En ellas, la dimensión espacial del proyecto neoliberal genera una serie de contradicciones y resistencias, en forma de movimientos sociales urbanos, que pueden ser interpretados bajo la consideración de su constitución como clases sociales. El barrio de Poblenou, en Barcelona, ha sido objeto de atención y transformación urbana desde hace décadas. Este artículo, como resultado de una investigación etnográfica de largo alcance enfocada en este tipo de procesos, realiza un análisis materialista de las acciones de grupos que históricamente han luchado contra formas específicas de desposesión en el entorno urbano particular que supone la capital de Cataluña.

PALABRAS CLAVE: Movimientos sociales; Lucha de clases; Barrios; Neoliberalismo.

ABSTRACT: Today, the processes of capital accumulation maintain an important role in cities. The spatial dimension of the neoliberal project generates its own contradictions as well as resistance in the form of urban social movements that can be interpreted under the consideration of their constitution as social classes. The neighborhood of Poblenou, in Barcelona, has been the subject of attention and urban transformation for decades. This article, as a result of a long ethnographic research focused in these dynamics, analyses from a materialist approach the involvement of those groups that historically have struggled against specific forms of dispossession and have formed social classes in this particular urban environment in the capital of Catalonia.

KEYWORDS: Social Movements; Class Struggle; Neighborhood; Neoliberalism.

Copyright: $\odot 2021$ CSIC. Este es un artículo de acceso abierto distribuido bajo los términos de la licencia de uso y distribución Creative Commons Reconocimiento 4.0 Internacional (CC BY 4.0).

1 Correo electrónico: joseamansilla@hotmail.com. ORCID iD: <https://orcid.org/0000-0002-1455-2928>. 


\section{BREVE INTRODUCCIÓN ETNOGRÁFICA}

El sábado 1 de febrero de 2020, la plataforma \#EnsPlantem, veïns en perill d'exinció había convocado a una cercavila contra la gentrificació que partiría desde el popular Parque de Can Felipa, junto al Centro Cívico del mismo nombre. Aunque el destino de la misma no se concretaba en los carteles y afiches que venían circulando desde días antes, la iniciativa parecía finalizar en algún tipo de comida popular, ya que los mensajes distribuidos desde \#EnsPlantem añadían la necesidad de llevar "plat i got». Si bien la cita era a las 11.30 h. -de la mañana- a las 11.40 h., la acción todavía no había comenzado y unas cuarenta personas esperaban y charlaban, conformando pequeños grupos, en el punto de encuentro. Miembros de la organización sostenían una pancarta que contenía únicamente 3 palabras, FORA LA MACRORESI, en referencia a la nueva residencia de lujo para estudiantes, con capacidad para hasta 752 plazas y precios superiores a mil euros, que estaba prevista construirse en el extremo nordeste del barrio ${ }^{2}$. Intrigado por la dirección de la manifestación, me dirigí a Roger, uno de los activistas y organizadores de la marcha,

«La idea es coger Camí Antic de València en dirección a la Macroresi. Luego ocuparemos el solar. Lo tenemos todo preparado. Organizaremos diferentes eventos, comidas populares, conciertos y demás, durante todo el fin de semana. La idea es acampar allí [...] Hemos estado pendientes y ya tenemos gente por la zona. Sabemos que han cambiado los guardias de seguridad; ha habido momentos en que ha habido hasta 2 de ellos. Creo que nos vigilan, pero es normal porque venimos anunciando esta mani desde hace tiempo». (Dialogo informal 1, Febrero 2020)

Justo a las 12.00 h.- la manifestación se puso en camino. Al final, la propuesta había logrado concentrar hasta cien personas que enfilaron, detrás de la pancarta, hacía el cercano Camí Antic de València. Durante todo el recorrido, que transcurrió entre esta calle, la Avenida Diagonal y las calles Josep Pla, Pallars, Agricultura y, finalmente, Pere IV, otros movimientos y colectivos, tanto del Poblenou como de barrios adyacentes -como el Sindicat

2 Para más información ver: <https://www.metropoliabierta.com/distritos/sant-marti/residencia-lujo-revoluciona-poblenou_22963_102.html>.
d'Habitatge de la Verneda i la Paü-, se fueron sumando al grupo inicial.

La marcha realizó el trayecto deteniéndose, por escaso tiempo, a las puertas del Hotel Vincci BIT, hasta llegar al que, inicialmente, parecía ser el destino final: el solar de la futura residencia. Sin embargo, a escasos metros de la puerta, pues el terreno se encuentra casi completamente amurallado, Rosa, otra de las activistas más reconocidas de \#EnsPlantem, se acercó a la altura de la manifestación en la que me encontraba para comentar que «las obras han comenzado. Han arrancado los árboles como forma de evitar la previsible ocupación del solar» (Diálogo informal 2, Febrero 2020). Efectivamente, al llegar a la entrada del recinto hasta 3 guardias de seguridad se encontraban situados en la puerta cerrada a cal y canto. En ese momento, los manifestantes comenzaron a correar consignas como «Esta residencia es una indecencia», «Especuladors fora del barri» o «El barri no està en venda, el barri es defensa» (ver Figura 1). En ese momento, uno de los activistas cogió un megáfono para denunciar el impacto que para el tejido social del barrio podía tener la apertura de un establecimiento de este tipo, así como anunciar los próximos movimientos, esto es, continuar hacía un solar próximo a Ca l'Isidret, un Casal de barrio recién inaugurado, donde, según comentarios del activista, "allí sí que ocuparemos».

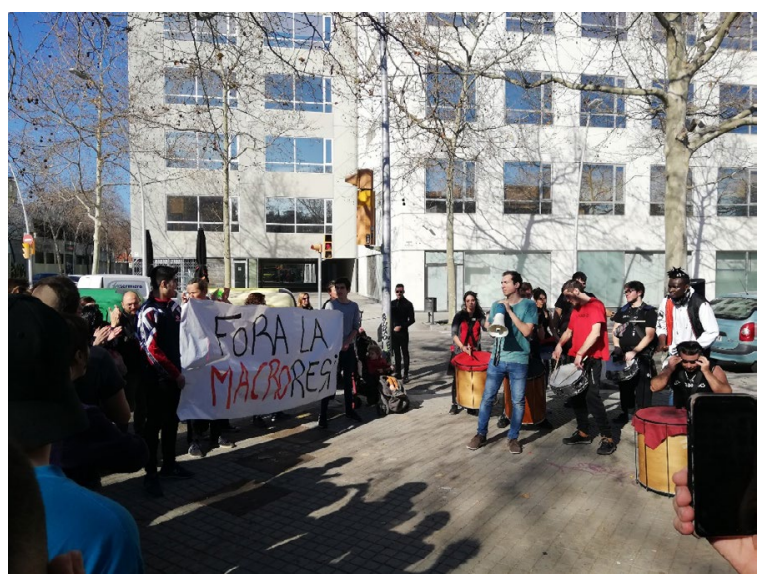

FIGURA 1.- Manifestación a las puertas del solar de la MACRORESI. Fuente: José Antonio Mansilla 2020.

3 De hecho, miembros de este Sindicato de Vivienda, a lo largo del recorrido, estuvieron distribuyendo unos panfletos en los cuales convocaban a una concentración con el objeto de detener un desahucio que se produciría el día 5 de febrero. Curiosamente, el propietario de la vivienda objeto de denuncia es la Generalitat de Catalunya, la cual cuenta con un parque público a través del Institut Català de Sól (INCASOL). 
A las 13.35 h. la manifestación llegaba al solar señalado, entrando libremente y desplegándose por el terreno; comenzaba su ocupación y la puesta en marcha del denominado Hort Fortaleça, un nuevo huerto urbano autogestionado en el Poblenou.

Este breve relato etnográfico supone un ejemplo más de los efectos del giro empresarialista (Brenner, Peck y Theodore 2015; Harvey 1989 y 2001), así como la contradicciones y resistencias, que genera la gestión de las ciudades desde mediados de los años 70 del pasado siglo; hecho que supuso la entrada de las fuerzas del mercado en aspectos que, hasta ese momento, habían permanecido bajo la esfera de la intervención pública. En el caso del siempre escaso suelo y la vivienda, la retirada del papel mediador del Estado y las posteriores políticas desreguladoras y liberalizadoras, acabaron por convertir estos elementos en auténticos depósitos de valor, un segundo circuito (Lefebvre 1972) que superaría, finalmente, la generación de plusvalías de la producción industrial típica del fordismo, mientras ésta era trasladada a la periferia del sistema mundo (Osorio 2015; Wallerstein 2005). Este avance hacia un modelo social y económico más flexible ha generado respuestas sociales específicas. Si los años 60 y 70 fueron testigos de la aparición de los denominados movimientos sociales urbanos (MSU) (Castells 1974 y 1983) como referentes de las demandas de consumo colectivo, las últimas décadas del siglo XX y las primeras del XXI han visto como estos movimientos se han adaptado a las características propias del neoliberalismo (Kling y Kosner 1990; Nicholls 2008; Thorn 2012; Feixa 2009 y 2013; Mansilla 2015a), de forma que han aparecido nuevas formas de organización, más flexibles y democráticas y menos jerárquicas, acordes a la nueva realidad que viven las ciudades y en las que es posible ver un componente de clase a la hora de realizar una aproximación desde las ciencias sociales.

\section{METODOLOGÍA E HIPÓTESIS}

El objetivo principal del presente artículo es, a través de una amplia investigación de carácter etnográfico, mostrar cómo la transformación residencial y productiva, la terciarización, de gran parte de la antigua área industrial del Poblenou (Barcelona), ha inducido la aparición de una contestación social que se ha articulado, específica y temporalmente, en función de la intensidad y características propias de esta metamorfosis. De este modo, en primer lugar se presentará la metodología seguida durante la investigación para, inmediatamente, marcas las líneas teóricas generales que vincularían la actuación de los movimientos sociales con las especificidades espaciales del neoliberalismo. A continuación, se trazará un recorrido por la historia de los principales proyectos de reforma urbana del barrio, donde aparecerán amplias referencias al trabajo de campo. Para finalizar, se señalarán una serie de conclusiones y propuestas teóricas para futuros análisis sobre procesos similares.

Tal y como se ha mencionado, se trata de un trabajo etnográfico que, como no podía ser de otra manera, se ha valido de una metodología principalmente cualitativa (Corbetta 2003; Hammersley y Atkinson 2007). Sin embargo, ésta se ha visto complementada con otra de tipo cuantitativo con el objetivo de realizar una triangulación metodológica (Creswell 2003) en un intento de apuntalar al máximo la proposición inicialmente presentada. De este modo, el primer paso a la hora de llevar a cabo el proceso etnográfico fue la realización de una revisión en profundidad de bibliografía relevante relacionada con el tipo de dinámica estudiada, no solo libros o artículos vinculados, sino también noticias, declaraciones, páginas webs y la participación en redes sociales de los principales actores involucrados. Estos aparecerán referenciados conforme vayan siendo útiles a la argumentación planteada.

El trabajo de campo se ha venido desarrollando, de manera intermitente, entre diciembre de 2012 y febrero de 2020; más de ocho años durante los cuales se ha hecho provisión de gran cantidad de datos y se ha participado en numerosos eventos. Las principales herramientas utilizadas han sido la observación participante, la celebración de entrevistas semiestructuradas, así como la participación en conversaciones y diálogos informales con algunos de los protagonistas. De este modo, en total se ha asistido a 13 asambleas y comisiones diversas, seis manifestaciones, tres de las cuales acabaron con la ocupación de equipamientos y solares públicos, y dos fiestas barriales. Además, se han llevado a cabo 21 entrevistas a diferentes participantes, se han mantenido seis diálogos informales y, por último, se han transcrito dos debates mantenidos a través de las redes sociales. Todas ellas han sido protagonizadas, principalmente, por miembros de la Assemblea Social del Poblenou (ASP9), el Ateneu la Flor de Maig, Fem Rambla, la Comissió d'Habitatge del Poblenou (HP9), la Taula Eix Pere IV y la Plataforma \#EnsPlantem, veïns en perill d'extinció, así como por integrantes de algunos de los huertos urbanos autogestionados del barrio, so- 
cios y Junta Directiva de la Associació de Veïns i Veïnes del Poblenou (AVVP9), trabajadores de antiguas fábricas del barrio, voluntarios del Arxiu Històric del Poblenou $^{4}$, activistas de antiguos movimientos y vecinos $y$ vecinas sin vinculación a colectivos o entidad alguna. Con el objetivo de evitar repeticiones, se han seleccionado los extractos más representativos de las entrevistas y los acontecimientos observados.

\section{LA DIMENSIÓN ESPACIAL DEL NEOLIBERALISMO Y LA CUESTIÓN DE LAS CLASES SOCIALES}

Las actuales dinámicas de transformación espacial pueden ser vistas como formas particulares del proceso de reestructuración vivido por el Estado bajo el neoliberalismo (Brenner y Theodore 2002). Entre sus rasgos distintivos encontraríamos el hecho de que su desarrollo está en marcha, es decir, estaríamos ante un paisaje donde instancias locales de reestructuración y resistencia se encuentran fuertemente determinadas por corrientes y contracorrientes institucionales e ideológicas aun en movimiento (Peck y Theodore 2012), incluso más de treinta años después de su popularización general.

A nivel urbano, este conjunto de prácticas «político-económicas» (Harvey 2005: 8) se caracterizaría, entre otros elementos, por considerar la ciudad como un espacio privilegiado para el despliegue del libre desarrollo de las capacidades e iniciativas empresariales, así como para la mercantilización de sus diferentes ámbitos de producción y reproducción social. Siguiendo a Harvey (2004), las urbes de todo el globo se aparecerían, de este modo, como localizaciones ideales para el despliegue de un nuevo tipo de imperialismo. En ellas, la esfera social habría devenido esfera productiva, de forma que no solo acogería los acontecimientos principales de la vida urbana, sino que, además, organizaría esa misma vida al ser objeto de intervención por parte de las relaciones de producción capitalista (Herin 1982).

4 El Ateneu la Flor de Maig es un centro social autogestionado del barrio, Fem Rambla fue una iniciativa vecinal puesta en marcha para exigir una mayor participación en los proyectos de reforma urbana, la Comissió d'Habitatge del Poblenou (HP9) es un colectivo de ayuda mutua y denuncia vinculado a los procesos de desahucio que viven algunas familias del barrio y el Arxiu Històric es una entidad sin ánimo de lucro que persigue velar por la memoria colectiva del Poblenou. El resto de movimientos serán presentados más adelante conforme vayan apareciendo en el relato etnográfico.
El neoliberalismo «explota y produce» (Brenner, Peck y Theodore 2015: 217) las diferencias socio-espaciales, generando, al mismo tiempo y como no podía ser de otra manera debido al carácter totalizador del capitalismo como sistema (Marx 1992), una respuesta por parte de los grupos sociales objeto de explotación. Esta dimensión urbana de la explotación capitalista ya fue destacada por Marx en La Guerra Civil en Francia (1871) ${ }^{5}$ y posteriormente recuperada, en diversas obras, por Henri Lefebvre, el cual, en un libro dedicado al estudio de la Comuna de París, La Proclamation de la Commune (1965), señalaría que los movimientos de tipo revolucionario acaban por asumir, en la mayoría de las ocasiones, una dimensión urbana que, finalmente y siguiendo a $E$. P. Thompson (2012), acabarían por conformar clases sociales. Este carácter de clase de los movimientos sociales urbanos sigue siendo objeto de investigación por parte de los estudios urbanos críticos ${ }^{6}$. En su libro Ciudades Rebeldes, David Harvey (2012) recoge, precisamente, el testigo de Lefebvre en obras como El derecho a la ciudad (1976) y Espacio y política. El derecho a la ciudad II (1976) y subraya cómo la clase obrera estaría constituida por «trabajadores urbanos de muy diversos tipos y no sólo de fábrica, que constituyen [...] una formación de clase muy diferente» $(2012: 11)^{7}$.

No obstante, autores como George Steinmetz han señalado los límites de una aproximación marxista al

5 «La Comuna [...] condonó los pagos de alquiler de viviendas desde octubre de 1870 hasta abril de 1871, abonando a futuros pagos de alquileres las cantidades ya pagadas, y suspendió la venta de objetos empeñados en el Monte de Piedad de la ciudad». (Marx 1971: 6)

6 Como se ha mencionado con anterioridad, Manuel Castells, a lo largo de la década de los 60 y 70 del pasado siglo XX, introdujo a la sociología urbana en el estudio de este tipo de movimientos. No obstante, a partir de la publicación de The city and the grasroots. A cross-cultural theory of urban social movements (1983), abandonaría la inicial orientación marxista de libros como La cuestión urbana (1974).

7 Cabe recordar, llegados a este punto, que el Capítulo LII del Libro III de El Capital, dedicado precisamente a las clases sociales, permaneció inacabado por Marx (2017). Este hecho es destacado por Sven-Eric Liedman (2020) en su obra Karl Marx, Una biografía, donde señala que el filósofo alemán siempre estuvo preocupado por realizar una correcta definición del término clase, algo que pudo dejar para el final porque, precisamente, no sabía cómo abarcar. Sin embargo, el primer párrafo de dicho capítulo señala la existencia de 3 clases: los asalariados, los capitalistas y los terratenientes, defiendo a estos últimos como los que poseen «rentas del suelo» (Marx, 2017), lo que nos aporta información interesante a la hora de definir los movimientos sociales de ámbito urbano como referentes de la lucha de clases enfrentados a la apropiación de plusvalías. 
análisis de los movimientos sociales. Para este sociólogo de la Universidad de Michigan, «aunque estos movimientos se han opuesto vigorosamente a muchas de las fuerzas de poder, propiedad y privilegio que los antiguos movimientos socialistas vinculados al trabajo hicieron, la forma en que se lleva a cabo esta oposición se diferencia en casi cada aspecto» (1994: 177). A esto, Steinmetz añade el hecho de que los actuales movimientos sociales tratan de marcar sus diferencias con otras organizaciones, como los partidos políticos tradicionales, además de actuar reactivamente, tal y como ya señalara en su momento Castells (1983), «pretendiendo prevenir las cosas antes de que sucedan» (Steinmetz 1994: 178), y sin contar con una verdadera agenda de futuro. Entre las características con las que Steinmetz define a los movimientos sociales se encuentra, además, el hecho de que éstos se centran en cuestiones vinculadas a la «autonomía, identidad, autorealización y cambios de vida cualitativos, antes que en el reparto de los recursos y beneficios materiales divisibles» (Ibid), con lo que parece destacar el carácter más cultural que material de los objetivos y reivindicaciones de los movimientos sociales y, por tanto, relegar su posible carácter de clase.

Esta crítica de Steinmetz, ciertamente frecuente, a las particularidades de los movimientos sociales ha sido contestadas por autores como Colin Baker (2013) que, en su análisis de la relación entre lucha de clases y movimientos sociales, recuerda que las luchas contra la opresión vinculada al racismo, el nacionalismo, la etnicidad, la religión o el género, no son distintas ni opuestas a la lucha de clases, sino que son mutuamente interdependientes y conforman parte de la lucha de los movimientos sociales contra el capitalismo considerado como totalidad. Es más, si como señalara Poulantzas el Estado, en sus diferentes formas y niveles, supone la "condensación de una relación de fuerzas definidas precisamente por la lucha» (1979: 147), el papel de los movimientos sociales se aparecería como determinante a la hora de generar cambios en los diferentes ámbitos de la vida social bajo su órbita. De esta forma, tal y como señalara Jessop (1990), las dinámicas y formas del capitalismo como sistema de apropiación de la plusvalía cambian constantemente, evolucionado desde determinadas estrategias de acumulación hasta llegar a proponer proyectos hegemónicos en su desarrollo como modo de producción. Es decir, no nos referimos únicamente a elementos objetivos relacionados con el funcionamiento del capital, sino a cómo se enfrenta, desde determinados sectores sociales «el intento de la clase capitalista por imponer su orden so- cial» (Cleaver 2000: 76). Bajo esta óptica, la cuestión material y la cultural no pueden más que ir de la mano, siendo indistinguibles en sus objetivos.

En este sentido, Nielsen y Cox proponen una definición de movimientos sociales más amplia cuando proponen a éstos como un «proceso en el cual un grupo social específico desarrolla un proyecto colectivo de actividades especializadas centradas en una racionalidad - una forma particular relacionada con el mundo social de crear sentido sobre el mismo - que intenta cambiar o mantener una estructura dominante de capacidades y necesidades atrincheradas, en parte o totalmente» (2013: 65-66). Este tipo de práctica puede derivar, también, en sujeto, es decir, en conciencia propia, como cuando se crean alianzas para resistir los intentos de privatización de determinados servicios básicos (Nielsen y Cox 2013) o cuando se rechaza de manera directa la transformación de un barrio o un proyecto urbanístico considerado inadecuado por el movimiento, como intentaremos mostrar en el presente texto.

Finalmente, esta concepción de los movimientos sociales como elementos conformantes de una lucha general contra el capitalismo estaría en línea con la visión de Erik Olin Wright (2018) en su aproximación a las clases como construcciones sociales determinadas a partir de dinámicas de explotación y dominación. Para Wright, estas relaciones de explotación/ dominación acabarían por beneficiar a aquellas clases capaces de controlar y restringir el acceso a los recursos de las clases sociales no dominantes. En el caso de los movimientos sociales analizados, esto se vería plasmado en su lucha contra la exclusión de la vida urbana, en definitiva, de su derecho a la ciudad (Lefebvre op. cit.).

\section{HI VA HAVER UN TEMPS EN QUE AL POBLENOU HI HAVIEN FABRIQUES ${ }^{8}$}

El barrio del Poblenou forma actualmente parte del Distrito de Sant Martí, una de las diez áreas político-administrativas en las que se encuentra dividida la ciudad. Sin embargo, a mediados del siglo XIX, el área era conocida como Icària o Llacuna y formaba parte del municipio de Sant Martí de Provençals.

8 «Hubo un tiempo en que en el Poblenou había fábricas». Traducción propia del catalán. Esta frase se ha tomado prestada del documental Poblenou, de la fàbrica al gratacel, de Produccións la Llacuna. Para más información: <https://www.youtube.com/watch?v=J1usCfr5xXY>. 
Por su localización fuera de las murallas de la ciudad, la disponibilidad de agua, su carácter plano y cercano al mar, la zona se convirtió en el emplazamiento ideal para acoger el primer desarrollo industrial de la capital catalana: el estampado de indianas (Fabre y Huertas 1980; Thomson 2003). Con el paso del tiempo, la zona se convirtió en la mayor concentración fabril de Catalunya, llegando a conocerse como el Mánchester Catalán, de forma acabaría por ser un núcleo urbano densamente poblado denominado Pueblo Nuevo o Poblenou (Marrero 2003; Tatjer 1973) ${ }^{9}$.

No obstante, y a semejanza de procesos similares ocurridos en otras partes de Europa y Estados Unidos, la zona comenzó a perder tejido industrial desde mediados del siglo XX: inicialmente, por su desplazamiento, en la propia ciudad, hacía la Zona Franca creada en 1965 tras un sinnúmero de vicisitudes y, luego, hacía otros municipios del área metropolitana de Barcelona, así como a otras regiones y países (Ortega 1977).

El desplazamiento de la actividad industrial hacía otros territorios liberó gran cantidad de suelo, el cual fue inicialmente ocupado, en su mayoría, por empresas de transporte y logística. Así mismo, el barrio también fue testigo de algunos procesos especulativos especialmente significativos que acabaron por generar respuestas populares organizadas. Es el caso del denominado Plan de la Ribera, en 1965. El Plan de la Ribera fue desarrollado por una sociedad de empresarios, La Ribera, S.A., que planteaba la construcción, dada la cercanía de la costa, de amplios complejos de viviendas de gama alta en terreros anteriormente destinados a compañías industriales. Entre sus objetivos estaba, además, la recuperación de las playas y el litoral; la eliminación del trazado ferroviario existente junto a la línea costera y el impulso a la terciarización

9 Isaac Marrero es autor de una interesante etnografía centrada en el caso de Can Ricart, importante recinto industrial del Poblenou que fue objeto de desalojo y posterior abandono en manos de distintas administraciones. Sin embargo, debido tanto al tratamiento exhaustivo ya realizado por Marrero, como a la falta de espacio en el presente texto, éste no ha sido tratado. Para más información ver: La fábrica del conflicto. Terciarización, lucha social y patrimonio en Can Ricart, Barcelona. Disponible en <https://www.tdx.cat/handle/10803/722> y el documental Destruir i construir. Història d'una fàbrica, disponible en <https://www.ccma.cat/tv3/alacarta/ el-documental/destruir-i-construir-historia-duna-fabrica/video/3959690/> productiva mediante la estimulación de la aparición de pequeño comercio junto a grandes centros comerciales. El Plan afectaba a un total de 234 hectáreas y tuvo dos características íntimamente relacionadas: fue el primer intento a gran escala de inversión capitalista de remodelación urbana con soporte institucional en el Estado español y, a la vez, supuso la primera gran contestación social frente a este tipo de iniciativas bajo el Franquismo (Solà-Morales et al 1974). Este segundo hecho tomó forma bajo la institucionalización de dos nuevos movimientos sociales en el barrio: la Associació de Veïns del Taulat y, dos años después, la Associació de Veïns del Poblenou (Fabré y Huertas Claveria 1976), los cuales podrían ser analizados bajo la lógica de los MSU de Castells (op. cit.). Finalmente, tanto la presión ejercida por el recién estrenado activismo vecinal, como la crisis de acumulación vivida por los países del Occidente capitalista durante la década del 70, unida a la situación de incertidumbre política del país, con la muerte del Dictador Franco y el periodo de Transición política hacía una democracia de corte parlamentario, acabaron por tumbar el proyecto. Sin embargo, y como veremos a continuación, esto no significó que el área se librara de dinámicas similares durante los siguiente años (Capel 2005; Mansilla 2015b).

\section{LOS JUEGoS OLíMPICOS DE 1992}

Aunque la idea se había venido gestando desde la década anterior (Moix 1994), en 1986 Barcelona fue proclamada en Lausanne (Suiza) sede de los Juegos Olímpicos (JJOO) del 92. Hoy día es posible afirmar que tal acontecimiento, junto a la celebración de la Exposición Universal de Sevilla y la proclamación de Madrid como Capital Europea de la Cultura, todo en el mismo año, representó, para España, su reconocimiento definitivo como país moderno, democrático y capitalista de finales de siglo. Para la capital catalana, este hecho supuso, asimismo, su espaldarazo definitivo como ciudad turística y de servicios (Mansilla y Milano 2018), además de una de las transformaciones socio-espaciales más importantes de su historia, la cual se llevó a cabo, precisamente, en el barrio del Poblenou y, en parte, en el mismo suelo que tendría que haber acogido el ya mencionado Plan de la Ribera. Se trató, en esta ocasión, de la transformación de unas 125 has., aproximadamente la mitad que el Plan, además de la construcción de unas 2.000 viviendas, en un principio destinadas a acoger a los deportistas olímpicos internacionales $y$, posteriormente, ser vendidas libremente en el mercado inmobiliario de la ciudad sin 
ningún tipo de protección pública, lo que ha dado lugar a lo que arquitecta y antropóloga Maria Gabriela Navas ha calificado, por su carácter elitista y exclusivo ${ }^{10}$, como una auténtica privatopía (2016). Los movimientos sociales del Poblenou no dejaron de movilizarse y expresar su preocupación y malestar por dicha transformación, ya que, como me señaló el actual miembro de la Associació de Veïns del Poblenou y, entonces, miembro de la Comissió Icària, Josep Lluis,

El espacio industrial que, no se [...], ya no se utilizaba para la industria, pues tenían un problema. Estaba calificado como solar industrial y no se podía construir. Entonces empezó a haber movimientos, que ya venían de lejos, desde el Plan de la Ribera, en los años 70 y pico, y que se materializaron en la Vila Olímpica [...] La Vila Olímpica fue la recalificación de suelo industrial a vivienda, así de duro, y por lo tanto fue un negocio redondo para los industriales que tenían las fábricas paradas y ya podían especular con el suelo (Entrevista 1, Mayo 2014).

La Comissió Icària fue un colectivo de carácter informal conformado por activistas de distinta condición y origen pero, sobre todo, por jóvenes provenientes de partidos políticos pertenecientes a la izquierda extraparlamentaria, que se organizaron, según Enric, otro de sus miembros, para

\begin{abstract}
discutir el plan estratégico de la ciudad, en el cual se veía claramente, sin hablar de gentrificación, el riesgo de los procesos de desplazamiento y cómo Poblenou formaría parte de esto [...] En las Juventudes Comunistas, donde yo militaba, en algún momento se discutió esto, pero sin oponerse [...], sin ningún análisis de clase de lo que suponía esto (Entrevista 2, Enero 2019).
\end{abstract}

Se trató, por tanto, de un movimiento que limitaba sus acciones a reuniones de análisis y acciones de difusión de su posición, aunque siempre bajo un fuerte componente de clase. La Comissió actuaría, de esta manera, como aquellos movimientos sociales desde abajo que propusiera Alf Gunvald Nielsen en su análisis marxista de este tipo de fenómeno, los cuales responderían a «infracciones o restricciones particulares [...] pero [que, a su vez] contienen la potencialidad contingente de un desarrollo expansivo hacía una forma más radical y transversal de

10 Hoy día, la Vila Olímpica es el 60 barrio por nivel de renta en la ciudad de Barcelona, con 58.562 euros/hogar según datos de la Agencia Española de Administración Tributaria (AEAT 2014). La ciudad cuenta con un total de 73 barrios. formas de acción colectiva» (2009: 113). En general, la oposición al mega-evento siempre fue escasa, liderada por pequeños grupúsculos de la izquierda política y parte de la intelectualidad local que veía los JJOO como un mito; un acontecimiento presentado como entelequia basado en cierto buenismo, algo que permitiría superar los conflictos "como si fuese un Congreso Eucarístico» (Moreno y Vázquez Montalbán 1991: 112).

\section{Hacía el Fòrum de les Cultures}

Las transformaciones del Poblenou continuaron durante los siguientes años, así como la articulación y respuesta ciudadana ante situaciones que fueron consideradas por ésta como contraría hacía los intereses de parte del barrio. Tras la construcción y venta de la Vila Olímpica, el mismo año de la celebración de los JJOO se puso en marcha el proyecto de construcción de lo que, entonces, fue denominado la Vila Olímpica 2 y que, en la actualidad, se conoce como Front Marítim, también en terreros recuperados del Plan de la Ribera. En total, unas 20,4 has. destinadas a siete bloques de 1.700 viviendas, de las cuales únicamente dos de los mismos tenía algún tipo de protección oficial. El resto se puso en manos de distintas inmobiliarias que vendieron las viviendas a precio de mercado. Aquí, la respuesta por parte de los movimientos sociales del barrio no estuvo destinada a protestar contra el tipo de barrio y de ciudad que se estaba construyendo, sino, más bien, a cuestiones de carácter práctico como la futura reubicación de los vecinos desalojados por la expropiación de los terrenos, a la reclamación de mayor participación en la toma de decisiones y a la construcción en el barrio de parques y otros tipos de infraestructuras. La continuidad de la Avenida Diagonal hasta la desembocadura del río Besòs, en el extremo nordeste de la ciudad, también supuso la transformación de 60 nuevas has., la expropiación de 730 viviendas y 500 pequeños negocios y empresas para construir, finalmente, 5.000 nuevos hogares que supusieron una nueva vuelta de tuerca en el aprovechamiento del anterior suelo industrial del barrio, así como su elitización.

Sin embargo, el colofón final de la expansión residencial del Poblenou se llevó a cabo con motivo de la celebración del Fòrum de les Cultures en 2004. Este nuevo mega-evento fue ideado por el Ayuntamiento de la ciudad en 1996 ante la imposibilidad de organizar una nueva Exposición Universal y tras el rechazo de las autoridades europeas a conceder a Barcelona su nombramiento 
como Ciudad Europea de la Cultura (Delgado 2007) ${ }^{11}$. Se trató de un proyecto desarrollado por capital completamente privado, el cual había adquirido, en 1990, 34 has. de terreno, 13 de las cuáles eran de propiedad semipública. El diseño giraba en torno a la construcción de un centro comercial, el complejo de ocio y consumo Diagonal Mar, de más de $87000 \mathrm{~m}^{2}$., que venía acompañado, en una segunda fase, por oficinas, hoteles y viviendas. Éstas fueron enclavadas en cinco supermanzanas, cada una con 400 unidades, además de un parque de unas 15 has., diseñado por el conocido arquitecto Enric Miralles. Volviendo a Josep Lluis,

En lo de Diagonal Mar no había afectados, porque eran terrenos de MACOSA ${ }^{12}$, y ahí si hicimos una crítica más al modelo de ciudad, también desde la Federació d'Associacions de Veïns i Veïnes de Barcelona (FAVB). Primero que se hiciera un parque con viviendas dentro, que esa promoción lo llevara una inmobiliaria de EEUU y que era una privatización del espacio, fue una crítica muy ideológica (Entrevista 1, Mayo 2004).

Tal y como el líder vecinal señala, sin embargo, la respuesta ciudadana no quedó únicamente restringida al ámbito del Poblenou, sino que abarcó la totalidad de la ciudad. Siguiendo a Harvey (2001), sería posible ver aquí un ejemplo de contestación dirigida al papel y la intervención del Estado en el proceso de acumulación en sí, en esta ocasión, mediante la provisión de bienes públicos y la infraestructura social y física necesaria para la reproducción del capital y trabajo. Esto supuso, además, la primera movilización popular espontánea no institucionalizada desde hacía décadas en Barcelona. Un ejemplo de las características de este nuevo tipo de movimiento fue la acción lúdico- reivindicativa Paterem el Fòrum, la cual se llevó a cabo en la mañana del día 18 de julio de 2004 cuando una flotilla compuesta por embarcaciones construidas con materiales de desechoen su mayoría

11 Para el antropólogo catalán Manuel Delgado, el Fòrum no va a ser más que un «dispositivo destinado a convertir la ciudad en un spot publicitario [...], una mera coartada para la promoción turística y comercial de la ciudad, una capa de falso moralismo pseudo-progresista con que cubrir las nuevas macro operaciones de especulación inmobiliaria, destinadas esta vez a explotar las grandes extensiones de suelo que abrió la prolongación de la Diagonal hasta el mar y la urbanización de la desembocadura del Besòs» (2007:41)

12 MACOSA, Maquinaria y Construcciones, S.A., empresa de construcción dedicada a la industria pesada.
(Figura 2.), salió de la cercana playa de la Mar Bella con el propósito de asaltar el recinto donde se desarrollaba el Fòrum. El objeto de la misma era, según palabras de Teo, activista y participante en Paterem,

Desenmascarar la hipocresía del Fòrum, la política del Gobierno de España en relación con la inmigración y la babosidad multicultural (sic) del evento. Entonces, la inmigración irregular comenzaba a llegar a las costas en patera desde el Norte de África, por eso llamamos a la acción Paterem (Entrevista 3, Marzo 2019) ${ }^{13}$.

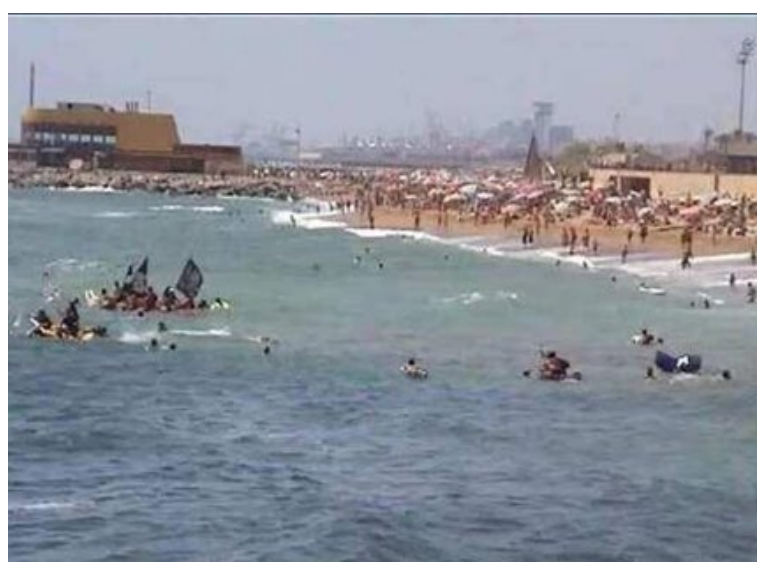

Figura 2. - Salida de la flotilla desde la Playa en el «Paterem el Fòrum» (2004). Fuente: desorgen.org

\section{DISTRITO TECNOLÓGICO, 15M Y HOTELES}

El último de los procesos de transformación urbana considerados en el presente capítulo es el conocido como Plan 22@. El desarrollo del Distrito Tecnológico 22@ constituyó, en su momento, la transformación más importante de la ciudad, ya que llegó a afectar a casi 116 hectáreas de suelo industrial. El nombre 22@ provenía de la recalificación de antiguos suelos calificados como industriales en el original Plan General

13 En el denominado Compromiso de Barcelona, presentado en la ceremonia de clausura del Fòrum, el día 26 de septiembre de 2014, se afirmaba lo siguiente: "Nosotros nos hemos encontrado, durante 141 días, para ver, presentar, debatir, escuchar, crear, disfrutar, disentir y acordar sobre las cosas que nos importan, presentando propuestas e iniciativas, adoptando acuerdos y compromisos, suscribiendo manifiestos y declaraciones, concretando proyectos a favor de la paz, de un desarrollo más sostenible y de la diversidad cultural». Este tipo de discursos era el que pretendían denunciar desde acciones como Paterem el Fòrum. Para más información ver: <http://www.fundacioforum.org/dossier_det. asp?id=2>. 
Metropolitano de 1976, los cuales recibían la etiqueta catastral 22a. El Plan se enmarcó dentro del documento de Modificación del Plan General Metropolitano del año 2000 (Ajuntament de Barcelona 2000), el cual se pretendía que fuera flexible ya que, como el mismo reconocía, la complejidad de las transformaciones, así como las operaciones que pretendía impulsar el Gobierno de la ciudad, junto a aquellas desarrolladas por la iniciativa privada, así lo exigían. Tal y como recogía su introducción, la finalidad del Plan era enfrentarse al reto de la nueva economía proponiendo al Poblenou como «la principal plataforma económica y tecnológica de Barcelona, Cataluña y España, en la perspectiva del Siglo XXI» (Ibid.: 14). Sin embargo, el paradigma de la nueva economía tuvo, finalmente, escasa presencia en el Poblenou, incumpliendo las expectativas levantadas desde un principio. Según un informe elaborado por el propio Ayuntamiento de la ciudad en 2016, quince años después de su lanzamiento, solo el $30 \%$ de las empresas instaladas en el 22@ se dedicaban a las nuevas tecnologías, siendo el 52,7\% compañías ya existentes que se trasladaban, desde otras partes de la ciudad, al nuevo Distrito, mientras que casi el 70\% fueron hoteles y compañías vinculadas a los seguros, los servicios financieros y el marketing (Ajuntament de Barcelona 2016).

Ahora bien, la contestación popular contra este nuevo proceso de «reproducción de la captura de las rentas urbanas monopólicas» (Charnock, Purcell y Ribera 2014: 15) fue, en un inicio, escasa. Así me lo señaló Pascual, Director de un Centro Cívico del barrio,

Hubo un movimiento contrario al 22@, pero sin tanta incidencia. Grupos concretos sin tanta discusión y polémica. Todos compramos la política que se nos vendió. No nos pareció un mal cambio. La actuación, en principio, era en zonas degradadas. Tenía que ir acompañada de una serie de cosas que no se han llevado a cabo. Nos hemos quedado con la parte chunga (sic) (Entrevista 4, Octubre 2013).

Sin embargo, tras el proceso de repolitización vivido en diversas ciudades a nivel español e internacional a raíz de las acampadas del 15M (Hughes 2011; Estalella y Corsin 2013), al que no fue ajena Barcelona (Antebi y Sánchez 2012), la situación cambió y los movimientos sociales, impulsados por la situación general, comenzaron a sumar participantes y activistas, abandonando su carácter generalmente minoritario y reactivo, y emprendiendo nuevas y ambiciosas acciones para exigir su derecho a la ciudad, en un ejemplo más de los «constantes procesos de cambio que se producen como resultado de la contienda entre grupos sociales dominantes y subalternos sobre la estructuración de necesidades y capacidades» (Nielsen, 2009). A esto se refería Rafel, educador social y activista barrial, cuando señalaba que «la onda de expansión de un 15M, [...] entró en el barrio» (Entrevista 5, Noviembre 2013). Movimientos del Poblenou surgidos a raíz de esta onda expansiva, tales como la Assemblea Social del Poblenou (ASP9), el Ateneu la Flor de Maig, Fem Rambla, la Comissió d'Habitatge del Poblenou (HP9), la Taula Eix Pere $\mathrm{IV}^{14}$ y otros, mediante la denuncia, las manifestaciones, la obstaculización de desahucios, el planteamiento de alternativas al ocio y el consumo mercantilizado, así como su participación en la ocupación de equipamientos, edificios y solares abandonados, comenzaron a cuestionar, entre otros elementos, la jerarquía de derechos y la preponderancia del valor de cambio sobre el valor de uso en una ciudad como Barcelona (Mansilla 2015a). Este boom de movilizaciones y articulación popular surgido a raíz del 15M ha llegado, de hecho, hasta nuestros días y puede ser analizado desde una perspectiva de clase, ya que éstas, como señalara Don Kalb,

No son ni lo trascendentes, ni lo coherentes que podamos desear, y menos aún se hallan empíricamente tan bien delineadas como a menudo nos las imaginamos [...]. Por el contrario, es más factible pensarlas a partir de la relación que las forman, para así especificar los mecanismos, los procesos y los cambios que tienen lugar en las relaciones sociales básicas. (1997: 20)

Entre los herederos de esos nuevos y más amplios movimientos sociales estaría también la plataforma \#EnsPlantem, veïns en perill d'extinció, iniciativa promovida por colectivos del barrio con el objetivo de hacer frente al proceso de turistificación del Poblenou (EnsPlantem 2016); dinámica generada, precisamente, por la proliferación de equipamientos turísticos en la zona 22@ y por la proyección de la ciudad, a nivel internacional, como nuevo y significado referente del turismo urbano (ver Figura 3.).

14 La Taula Eix Pere IV es una plataforma ciudadana formada per vecinos y vecinas, colectivos y cooperativas de los barrios del Poblenou reunidas con el objetivo de impulsar la reactivación social, cultural y económica del eje viario Pere IV (Taula Eix Pere IV 2014). 
De este modo, según Alicia, activista de la plataforma,

En cuatro o cinco años, sobre el turismo y otras cuestiones, lo que ha pasado ha sido un despertar colectivo frente a muchas cosas, o sea, cosas que en la intimidad de nuestras casas y con nuestros amigos lo comentábamos, decíamos 'hostia, tú, como están especulando. A mí me subieron el alquiler no sé cuántas veces y de forma muy bestia' Pero cuando lo decías, te miraban como diciendo '¿Quieres decir? Bueno no, pero busca [...] Y yo no, no, esto se está disparando, esto es una locura [...]' Hace diez años no te decían nada, pero en los últimos cuatro o cinco años la gente ha hecho un clic [...] Y no solo al tema de la vivienda, sino también al espacio público, a la especulación de la banca [...] a muchas cosas que yo creo que, o yo lo relaciono con el $15 \mathrm{M}$, para mí sí que tiene que ver, para mí sí que fue un momento de despertar colectivo y, en concreto, en el tema del turismo en el Poblenou. (Entrevista 6, Febrero 2017)

Las actividades de la plataforma \#EnsPlantem, dirigidas principalmente a luchar contra el incremento del precio de la vivienda, la privatización del espacio urbano del barrio y los cambios en el paisaje urbano del comercio local, consiguieron, entre otras cuestiones y como se ha comentado antes, situar la proliferación sin control de la actividad turística en el mapa de la opinión pública, así como que la normativa municipal dirigida a regular y planificar los equipamientos turísticos de la ciudad, considerara al barrio como zona de decrecimiento (BOPB 2017).

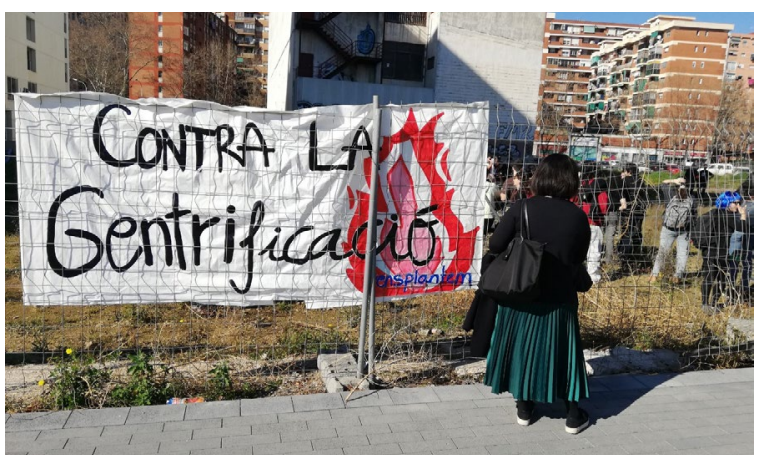

FIguRA 3.- Puesta en marcha del Hort Fortaleça (2020). Fuente: José Antonio Mansilla.

Sin embargo, las vicisitudes en torno al Distrito 22@ no habrían acabado. Durante el año 2017, el Ayuntamiento de la ciudad, en manos del izquierdista Barcelona en comú ${ }^{15}$ en coalición con el Partit dels Socialis-

15 El origen de Barcelona en comú se halla, precisamente, en movimientos sociales con fuerte presencia en la tes de Catalunya (PSC) ${ }^{16}$, decidió poner en marcha un proceso participativo denominado Repensem 22@. Se trataba de realizar un diagnóstico abierto e inclusivo que permitiera realizar una propuesta estratégica sobre el futuro de la zona (Repensem 22@ 2017). La metodología incluía dos bloques de participantes: por un lado la denominada Comissió Ampliada -un equipo técnico municipal junto a entidades vecinales- donde se encontraban movimientos sociales del barrio, como Taula Eix Pere IV, y la FAVB-, económicas y universitarias de la zona-, liderada por la Fundació BIT Habitat, $y$, por otro lado, un proceso participativo puramente popular. Ambos deberían retroalimentarse. Tal y como me comentó Robert, sociólogo y trabajador municipal:

Desde el Ayuntamiento se piensa que el urbanismo es demasiado importante para dejarlo en manos de los procesos participativos. Para los vecinos solo dejan el diseño de placitas [...] En torno al 22@, desde el barrio se ha actuado de forma más técnica, podría ser porque se ha asumido la derrota y no se cuenta con una masa crítica para llevarlo a la calle. Sin embargo, sí es verdad que propuestas elaboradas en los momentos álgidos de lucha contra el 22@, se han acabado asumiendo por movimientos actuales como la Taula Eix Pere IV. (Entrevista 7, Enero 2019)

El resultado final, el mencionado Pacte Cap a un Poblenou amb un 22@ més inclusiu i sostenible, objeto de las protestas descritas en la introducción del presente artículo, fue finalmente rechazado debido a la falta de acuerdo político.

\section{BREVES CONCLUSIONES Y PROPUESTAS FINALES}

En un mundo cada vez más urbano, las ciudades suponen el escenario ideal para las dinámicas de reproducción y circulación del capital, pero también para evidenciar sus contradicciones y mostrar la aparición de viejas y nuevas formas de movilización y, he aquí la propuesta, lucha de clases. El carácter de sistema total del capitalismo, así como las características especiales y espaciales del modelo neoliberal, enfrentan unas resistencias protagonizadas por unos movimientos sociales que ven como espacios anteriormente

ciudad como la Plataforma de Afectados por la Hipoteca (PAH), donde la actual alcaldesa fue, de hecho, portavoz del Observatori de Drets Econòmics, Socials i Culturals (DESC), entre otros.

16 El Partit dels Socialistes de Catalunya (PSC) abandonó el Gobierno municipal en el verano de 2017. 
destinados a la socialización, como las propias calles y las plazas de las ciudades, así como elementos con una importante función social, como la vivienda o los equipamientos y servicios públicos, acaban formando parte de los procesos de generación de plusvalías y de acumulación por desposesión (Harvey 2004). Estos movimientos, al ser generados mediante relaciones de explotación y dominación entre grupos unidos por intereses, a veces, opuestos, pueden ser analizados desde una perspectiva de clase ya que, volviendo a Harvey (2008: 24), «los excedentes [urbanos] son extraídos de algún sitio y de alguien, mientras que el control sobre su utilización habitualmente radica en pocas manos». De este modo, los fondos de inversión, las inmobiliarias, los intermediarios, los promotores, los hoteles, turoperadores y otras empresas turísticas y de la restauración, promovidos y congregados por el Estado bajo sus diferentes niveles administrativos, conformarían un conjunto social en busca de beneficios a partir de la ciudad y la vida urbana, mientras que, por otro lado, los vecinos y vecinas, antiguos usuarios de su espacioy ahora consumidores del mismo, son excluidos, desplazados, de esta vida y de su derecho a la ciudad. Es posible ver, así, cómo el punto inicial de muchas de las movilizaciones descritas se encuentra en "prácticas cotidianas desarrolladas en respuesta a necesidades, problemas y lugares específicos, fundamentadas materialmente en situaciones concretas y, por lo tanto, en un grupo específico» (Nielsen y Cox 2013), pero que pueden adquirir una articulación más abstracta y más subjetiva, en su conformación como intereses de clase, en cuanto se articulan con otros aliados situados en condiciones y espacios similares. Es aquello que Neil Smith denominó "salto de escala» (1993), es decir, aquellas estrategias que los actores y los grupos sociales ponen en marcha para escalar en sus demandas, escapando del particularismo militante localizado y ciertamente infructuoso en las luchas contra el capital inmobiliario (Harvey 2000).

En el caso del Poblenou, estas relaciones han conformado distintos movimientos a lo largo de su historia reciente, manifestando características distintas según el momento, el contexto y la oportunidad; han constituido clases sociales, o movimientos sociales con conciencia propia, en palabras de Nielsen y Cox (2013), a partir de los mecanismos y procesos de explotación capitalista sobre un barrio que, como no podía ser de otra manera, ha acabado por hacer aparecer fricciones externas e internas en grupos que, las más de las veces, no se representan a sí mismos bajo la etiqueta de clase.

El caso de Barcelona muestra, además, las limitaciones de la acción política realizada desde las instituciones democráticas pues, como se ha visto, la incapacidad de alcanzar acuerdos amplios por parte de fuerzas teóricamente al servicio de las clases populares de la ciudad, ha acabado por dar al traste con algunas iniciativas donde la participación ciudadana no solo ha sido activa, sino también pragmática con los resultados obtenidos. La celebración, en Mayo de 2019, de nuevas elecciones municipales dio como resultado una renovación del mandato de Barcelona en comú en coalición con el PSC. Estos cuatro años de mandato serán una ocasión excelente para observar y analizar cómo las fuerzas políticas podrían emprender iniciativas y medidas continuistas o diferentes y, también, cómo la respuesta popular acaba por articularse en torno a ellas.

\section{REFERENCIAS BIBLIOGRÁFICAS}

Ajuntament de Barcelona. 2000. Modificació del PGM per la renovació de les zones industrials del Poblenou - Distrito d'Activitats 22@BCN-. Barcelona: Ajuntament de Barcelona. Disponible en: <http://www3.amb.cat/normaurb2004/ Docs/Normes_mod/NUM-Barcelona-5.pdf>. Fecha de acceso: 12 feb. 2020 .

Ajuntament de Barcelona. 2016. 22@Barcelona, 2000-2015. El districte de la innovació de Barcelona. Disponible en <http:// www.ajuntamentbarcelonapremsa.info/download/22_15_ ANYS_CATALA_p.pdf>. Fecha de acceso: 12 feb. 2020.

Antebi, Andrés y José Sánchez. 2012. «Plazas fuertes: de Midan a la Plaça Catalunya: espacio público y revueltas populares contemporáneas». En Carlos Sevilla, Joseba Fernández, y Miguel Urban (eds.), Ocupemos el Mundo: 67-81. Barcelona: Icaria Editorial.

Brenner, Neil y Nik Theodore. 2002. "Cities and the Geographies of Actually Existing Neoliberalism». Antipode, 34: 349-79.

Baker, Colin. 2013. "Class Struggle and Social Movement». En Colin Baker et al, Marxism and Social Movements: 41-62. Leiden/Boston: Brill.

BOPB. 2017. Aprovació del Pla Especial Urbanístic d'Allotjaments Turístics (PEUAT). DIBA. Disponible en <https://bop.diba. cat/scripts/ftpisa.aspx?fnew?bop2017\&03/022017003806. pdf\&1>. Fecha de acceso: 12 feb. 2020.

Brenner, Neil, Jamie Peck y Nik Theodore. 2015. «Urbanismo neoliberal. La ciudad y el imperio de los mercados». En Observatorio Metropolitano de Madrid (eds.), El mercado contra la ciudad. Globalización, gentrificación y políticas urbanas: 211-244. Madrid: Traficantes de Sueños. 
Capel, Horacio. 2005. El modelo Barcelona: un examen crítico. Barcelona: Ediciones del Serbal.

Castells, Manuel. 1974. Movimientos sociales urbanos. Madrid: Siglo XXI.

Castells, Manuel. 1974. La cuestión urbana. Madrid: Siglo XXI.

Castells, Manuel. 1983. The City and the Grasroots. A Cross-Cultural Theory of Urban Social Movements. Berkeley: University of California Press.

Charnock, Greig, Theodore Purcell y Ramon Ribera-Fumaz. 2014. "City Of Rents: the Limits to the Barcelona Model Of Urban Competitiveness». International Journal of Urban and Regional Research, 38(1): 198-217.

Cleaver, Harry. 2000. Reading Capital Politically. Chico: AK Press.

Corbetta, Piergiorgio. 2003. Social Research: Theory, Methods and Techniques. Londres (UK), Thousand Oaks, Cal. (US): SAGE.

Creswell, John W. 2003. Research Design: Qualitative, Quantitative, and Mixed Method Approaches. Los Angeles, Cal. (US), Londres (UK): Sage Publications.

Delgado, Manuel. 2007. La ciudad mentirosa. Fraude y miseria del «modelo Barcelona». Madrid: Los libros de la Catarata.

EnsPlantem. 2016. "Nota de Premsa». La Flor de Maig. Disponible en <https://laflordemaig.cat/2016/06/nota-de- premsa-ensplantem-veins-en-perill-dextincio-05062016/>. Fecha de acceso: 12 feb. 2020

Estelella, Adolfo y Alberto Corsín-Jiménez. 2013. «Asambleas al aire. La arquitectura ambulatoria de una política en suspensión». Revista de Antropología Experimental, 13: 73-88.

Fabré, Jaume y Josep Maria Huertas Claveria. 1980. Tots els barris de Barcelona. Barcelona: Edicions 62.

Feixa, Carles, Inés Pereira y Jeffrey S. Juris. 2009. «Global Citizenship and the 'New, New' Social Movements: Iberian Connections». YOUNG, 17(4): 421-442. doi: <https://doi.org/10. $1177 / 110330880901700405>$

Feixa, Carles. 2013. "The \#spanishrevolution and Beyond». Fieldsights - Hot Spots, Cultural Anthropology online. Disponible en <http://culanth.org/fieldsights/68-the-spanishrevolution-and- beyond>. Fecha de acceso: 12 feb. 2020.

Hammersley, Martyn y Paul Atkinson. 2007. Ethnography: Principles in Practice. Londres-Nueva York: Routledge.

Harvey, David. 1989. «From Managerialism to Entrepreneurialism: The Transformation in Urban Governance in Late Capitalism». Geografiska Annaler. Series B, Human Geography, 71(1): 3-17. doi: <http://dx.doi.org/10.2307/490503>.

Harvey, David. 2000. Espacios de esperanza. Madrid: Akal.

Harvey, David. 2001. Espacios del capital. Hacia una geografía crítica. Madrid: Akal.

Harvey, David. 2004. El nuevo imperialismo. Madrid: Akal.

Harvey, David. 2005. Breve historia del neoliberalismo. Madrid: Akal.

Harvey, David. 2008. «El derecho a la ciudad». New Left Review, 53: 23-39.
Hughes, Neil. 2011. "Young People Took to the Streets and all of a Sudden all of the Political Parties Got Old: The 15M Movement in Spain». Journal of Social Movements, 10(4): 407-413.

Jessop, Bob. 1990. State Theory: Puting the Capitalist State in its Place. Cambridge: Poloty Press.

Lefebvre, Henri. 1965. La proclamation de la Commune. París; Galimard.

Lefebvre, Henri. 1978. El derecho a la ciudad. Barcelona: Península.

Lefebvre, Henri. 1972 La revolución urbana. Madrid: Alianza Editorial.

Herin, Robert. 1982. "Herencias y perspectivas en la geografía social francesa». Geo-Crítica: Cuadernos Críticos de Geografía Humana, 41: 26-33.

Kalb, Don. 1997. Expanding Class. Power and Everyday Politics in Industrial Communities. Durham (N.C.): Duke University Press.

Kling, Joseph y Prudence Posner (eds.). 1990. Class and Community: Theories of Activism in the Era Of Urban Transformation. Dilemmas of Activism: Class, Community and the Politics of the Local Mobilization. Philadelphia: Temple University Press.

Liedman, Sven-Eric. 2020. Karl Marx, Una biografía. Madrid: Akal.

Mansilla, José A. 2018. "Vecinos en peligro de extinción. Turismo urbano, movimientos sociales y exclusión socioespacial en Barcelona». Revista PASOS, 16(2): 279-296.

Mansilla, José A. 2014. «La Flor de Maig somos nosotros. Geografía urbana de la memoria en el Poblenou, Barcelona». Scripta Nova. Revista electrónica de geografía y ciencias sociales. Vol. XVIII, 493(05). doi: <http://dx.doi.org/10.1344/ sn2014.18.14976>.

Mansilla, José A. 2015a. "Movimientos sociales y apropiaciones colectivas en la Barcelona Post- 15M: El Papel de la Assemblea Social del Poblenou». Etnográfica 19(1): 77-97.

Mansilla, José A. 2015b. La Flor de Maig somos nosotros. Una etnografía de la memoria en el barrio del Poblenou, Barcelona. Tesis Doctoral. Barcelona: Universitat de Barcelona. Disponible en <https://www.tdx.cat/bitstream/handle/10803/352463/JAML_TESIS.pdf?sequence=1>. Fecha de acceso: 12 feb. 2020.

Mansilla, Jose A. y Claudio Milano. 2018. «Introducción a la ciudad de vacaciones. Apuntes sobre turismo y malestar social en Barcelona». En Claudio Milano y José A. Mansilla (coords.), Ciudad de vacaciones. Conflictos urbanos en espacios turísticos: 19-80. Barcelona: Pol- len Edicions.

Marrero, Isaac. 2003. «¿Del Manchester catalán al Soho Barcelonés? La renovación del barrio del Poble Nou en Barcelona y la cuestión de la vivienda». Scripta Nova. Revista electrónica de geografía y ciencias sociales. Vol. VII, 146(137). Disponible en a <http://www.ub.es/geocrit/sn/sn- 146(137). htm>. Fecha de acceso: 12 feb. 2020.

Marx, Karl. 1871. La Guerra civil en Francia. Disponible en <http://www.enxarxa.com/biblioteca/MARX\%20La\%20 
guerra\%20civil\%20en\%20Francia.pdf>. Fecha de acceso: 12 feb. 2020.

Marx, Karl. 1992. El Capital. Moscú: Editorial Progreso.

Marx, Karl. 2017. El Capital, Libro III. Madrid: Ed. Siglo XXI.

Moix, Llatzer. 1994. La ciudad de los arquitectos. Barcelona: Anagrama.

Moreno, Eduardo y Manuel Vázquez Montalbán. 1991. Barcelona, ¿a dónde vas? Barcelona: Ed. La Tempestad.

Navas Perrone, María Gabriela. 2016. «Utopía y privatopía en la Vila Olímpica de Barcelona. Impactos sociales de un barrio de autor». Tesis Doctoral. Barcelona: Universitat de Barcelona. Disponible en <https://www.tdx.cat/handle/10803/401430>. Fecha de acceso: 12 feb. 2020.

Nicholls, Walter. 2008. "The Urban Question Revisited: The Importance of Cities for Social Movements». International Journal of Urban and Regional Research. doi: <https://doi. org/10.1111/j.1468-2427.2008.00820.x>.

Nielsen, Alf Gunvald. 2009. "The authors and the actors of their own drama: Towards a Marxist theory of social movements». Capital and Class, 33: 109-139.

Nielsen, Alf Gunvald y Laurence Cox. 2013. "What Would a Marxist Theory of Social Movements Look Like?». En Colin Baker et al., Marxism and Social Movements: 41-62. Leiden/ Boston: Brill.

Ortega, Eloísa. 1977. «La Zona Franca de Barcelona. De Puerto Franco a Polígono Industrial». Revista de Geografía 11(1-2): 89-109.

Osorio, Jaime. 2015. «El sistema-mundo de Wallerstein y su transformación: Una lectura crítica». Argumentos 28(77): 131-154. México, D.F. Disponible en <http://www.scielo.org.mx/scielo. php?script=sci_arttext\&pid=S0187-57952015000100007\&lng=es\&tlng=es>. Fecha de acceso: 12 feb. 2020.

Peck, Jamie y Nik Theodore. 2012. «Reanimating Neoliberalism: Process Geographies of Neoliberalisation». Social An-
thropology/Anthropologie Sociale, 20(2): 177-185. <https:/ /10.1111/j.1469-8676.2012.00194.x>.

Poulantzas, Nicos. 1979. Estado, Poder y Socialismo. Madrid: Ed. Siglo XXI.

Repensem 22@. 2017. Repensem el 22@. Disponible en <https://www.decidim.barcelona/processes/Repensem22a>. Fecha de acceso: 12 feb.

Smith, Neil .1993. «Homeless/Global: Scaling Places». En Bird, J. et al. (eds.) Mapping the Futures: Local Cultures, Global Change: 87-119. Nueva York: Routledge.

Solà-Morales, Manuel et al. 1974. Barcelona, remodelación capitalista o desarrollo urbano en el sector de la ribera oriental. Barcelona: Ed. Gustavo Gili.

Steinmetz, George. 1994. «Regulation Theory, Post-Marxism, and the New Social Movements». Comparative Studies in Society and History, 36(1): 176-212.

Tatjer, Mercè. 1973. La Barceloneta del siglo XVIII al Plan de la Ribera. Barcelona: Los Libros de la Frontera.

Taula Eix Pere IV. 2014. Qui som? Disponible en <https://eixpereiv.org/la-taula/>. Fecha de acceso: 12 feb.

Thompson, Edward P. 2012. La formación de la clase obrera en Inglaterra. Madrid: Capitan Swing.

Thomson, J.K.J. 2003. "Transferencia tecnológica en la industria catalana: de las indias a la selfactina». Revista de Historia Industrial, 24: 13-49.

Thorn, Hakan. 2012. "In Between Social Engineering and Gentrification: Urban Restructuring, Social Movements and the Place Politics of Open Space». Journal of Urban Affairs Vol. 34(2): 153-168.

Wallerstein, Immanuel. 2005. Análisis de sistemas-mundo. Una introducción. México: Siglo XXI Editores.

Wright, Eric O. 2018. Comprender las clases sociales. Madrid: Akal. 